\title{
Mimicking the colourful wing scale structure of the Papilio blumei butterfly
}

Mathias Kolle 1,2 $_{1, \text { Pedro M. Salgard-Cunha }}$, Maik R. J. Scherer ${ }_{1}$, Fumin Huang, Pete Vukusic $_{3}$, Sumeet Mahajan 4 , Jeremy J. Baumberg ${ }_{1} \&$ Ullrich Steiner ${ }_{1}$

${ }^{1}$ Department of Physics, Cavendish Laboratory, University of Cambridge, $C B 3 \mathrm{OHE}$, UK

${ }^{2}$ Nanoscience Centre, University of Cambridge, Cambridge, CB3 OFF, UK

${ }^{3}$ School of Physics, Stocker Road, Exeter, EX4 4QL, UK

${ }^{4}$ School of Chemistry, University of Southampton, Southampton UK SO17 1BJ.

E-mail: S.Mahajan@soton.ac.uk

Please cite this paper as:

Nature Nanotechnology, 2010, (5) 511-515

The publisher's version of this paper is available here:

http://dx.doi.org/10.1038/NNANO.2010.101

\section{Related articles by Dr Sumeet Mahajan can be found below: \\ Daniel O. Sigle, Elaine A. Perkins, Jeremy J. Baumberg and Sumeet Mahajan, (2013) Reproducible deep-UV SERRS on aluminium nanovoids. The Journal of Physical Chemistry Letters (doi:10.1021/jz4004813).}

James T. Hugall, Jeremy J. Baumberg, and Sumeet Mahajan, (2012) Disentangling the peak and background signals in surface-enhanced raman scattering. The Journal of Physical Chemistry C, 116, (10), 6184-6190. (doi:10.1021/jp3002977).

Robin M. Cole, Sumeet Mahajan and Jeremy J. Baumberg, (2009) Stretchable metal-elastomer nanovoids for tunable plasmons. Applied Physics Letters, 95, (15), 154103-154103. (doi:10.1063/1.3247966).

Sumeet Mahajan, Robin M. Cole, Bruno F. Soares, Suzanne H. Pelfrey, Andrea E. Russell, Jeremy J. Baumberg and Philip N. Bartlett, (2009) Relating SERS intensity to specific plasmon modes on sphere segment void surfaces. The Journal of Physical Chemistry C, 113, (21), 9284-9289. (doi:10.1021/jp900661u).

Sumeet Mahajan, James Richardson, Tom Brown and Philip N. Bartlett, (2008) SERS-melting: a new method for discriminating mutations in dna sequences. Journal of the American Chemical Society, 130, (46), 15589-15601. (doi:10.1021/ja805517q). 


\title{
Mimicking the colourful wing scale structure of the Papilio blumei butterfly
}

\author{
Mathias Kolle ${ }^{1,2}$, Pedro M. Salgard-Cunha ${ }^{1}$, Maik R. J. Scherer ${ }^{1}$, Fumin Huang ${ }^{1}$, \\ Pete Vukusic ${ }^{3}$, Sumeet Mahajan ${ }^{1}$, Jeremy J. Baumberg ${ }^{1}$ \& Ullrich Steiner ${ }^{1}$ \\ ${ }^{1}$ Department of Physics, Cavendish Laboratory, University of Cambridge, Cambridge, CB3 OHE, UK \\ ${ }^{2}$ Nanoscience Centre, University of Cambridge, Cambridge, CB3 OFF, UK \\ ${ }^{3}$ School of Physics, Stocker Road, Exeter, EX4 4QL, UK
}

(Dated: April 19, 2010)

The brightest and most vivid colours in nature arise from the interaction of light with surfaces that exhibit periodic structure on the micro- and nanoscale. In the wings of butterflies, for example, a combination of multi-layer interference, optical gratings, photonic crystals and other optical structures gives rise to complex colour mixing. While the physics of structural colours is well understood, it remains a challenge to create artificial replicas of natural photonic structures ${ }^{1-3}$. Here we use a combination of layer deposition techniques, including colloidal self-assembly, sputtering and atomic layer deposition, to fabricate photonic structures that mimic the colour mixing effect found on the wings of the Indonesian butterfly Papilio blumei. We also show that a conceptual variation to the natural structure leads to enhanced optical properties. Our approach offers improved efficiency, versatility and scalability compared with previous approaches ${ }^{4-6}$.

The intricate structures found on the wing scales of butterflies are difficult to copy, and it is particularly challenging to mimic the colour mixing effects displayed by P. blumei and Papilio palinurus ${ }^{7,8}$. The wing scales of these butterflies consist of regularly deformed multilayer stacks that are made from alternating layers of cuticle and air, and create intense structural colours (Fig. 1). Although the $P$. blumei wing scales have previously been used as a template for atomic layer deposition ${ }^{9}$ and solgel techniques ${ }^{10,11}$, such an approach is not suitable for accurate replication of the internal multilayer structure on large surface areas.

The bright green coloured areas on Papilio blumei and Papilio palinurus wings result from a juxtaposition of blue and yellow-green light reflected from different microscopic regions on the wing scales. Light microscopy reveals that these regions are the centres (yellow) and edges (blue) of 5-10 $\mathrm{mm}$ wide concavities, clad with a perforated cuticle multilayer ${ }^{7}$ (Fig. 1d,e).

For normal light incidence, the cuticle-air multilayer shows a reflectance peak at a wavelength of $\lambda_{\max }=$ $525 \mathrm{~nm}$, which shifts to $\lambda_{\max }=477 \mathrm{~nm}$ for light incident at an angle of $45^{\circ}$. Light from the centre of the cavity is directly reflected, while retro-reflection of light incident onto the concavity edges occurs by double re- flection off the cavity multilayer (Fig. 1g). This double reflection induces a geometrical polarisation rotation ${ }^{12}$. If light, that is polarised at an angle $\psi$ to the initial plane of incidence, is retro-reflected by the double bounce, it will pick up a polarisation rotation of $2 \psi$ and the intensity distribution through collinear polarisers is therefore given by $\cos ^{2}(2 \psi)$. This leads to an interesting phenomenon: When placing the sample between crossed polarisers, light reflected off the centres of the cavities is suppressed, whereas retro-reflected light from four segments of the cavity edges is detected ${ }^{12,13}$ (Fig. 1d, right).

In microstructures without this double reflection, both the colour mixing and polarisation conversion are absent. This is the case for scales of Papilio ulysses, a relative of $P$. blumei and $P$. palinurus, which has considerably shallower concavities ${ }^{8}$ that cannot retroreflect incident light, and thus shows only a more conventional shimmering blue/violet colour.

The replication of natural photonic structures is useful for the creation of model systems to better understand structural colour in nature. Here, we demonstrate the replication of the periodically shaped multilayer structure of the Papilio butterfly scale in only five steps (Fig. 2). Avoiding the full structural complexity based on alternating solid cuticle and cuticle-pillar-supported air layers, we aim primarily to reproduce its optical characteristics. The multilayer structure of the artificial mimic is instead realised with two solid inorganic constituents. This results in a much simpler structural design but with the appropriate materials, structurally simplified replicas with authentic optical performance are realised.

In order to create regularly arranged concavities of appropriate dimensions, polystyrene colloids of $5 \mu \mathrm{m}$ diameter are assembled on a gold-coated silicon substrate. Subsequently, a $2.5 \mathrm{~mm}$ thick layer of platinum or gold is electrochemically grown into the interstitial space between the colloids, creating a negative replica ${ }^{14-16}$. Ultrasonication of the sample in dimethylformamide or acetone removes the colloids, resulting in a template of hexagonally arranged metal concavities. A $20 \mathrm{~nm}$ thick carbon film is sputtered onto the gold surface. Finally, a conformal multilayer of thin quarter-wave titania and alumina films is grown by atomic layer deposition ${ }^{17}$. The carbon layer between the gold (or platinum) and the multilayer stack adsorbs light that passes through the mul- 

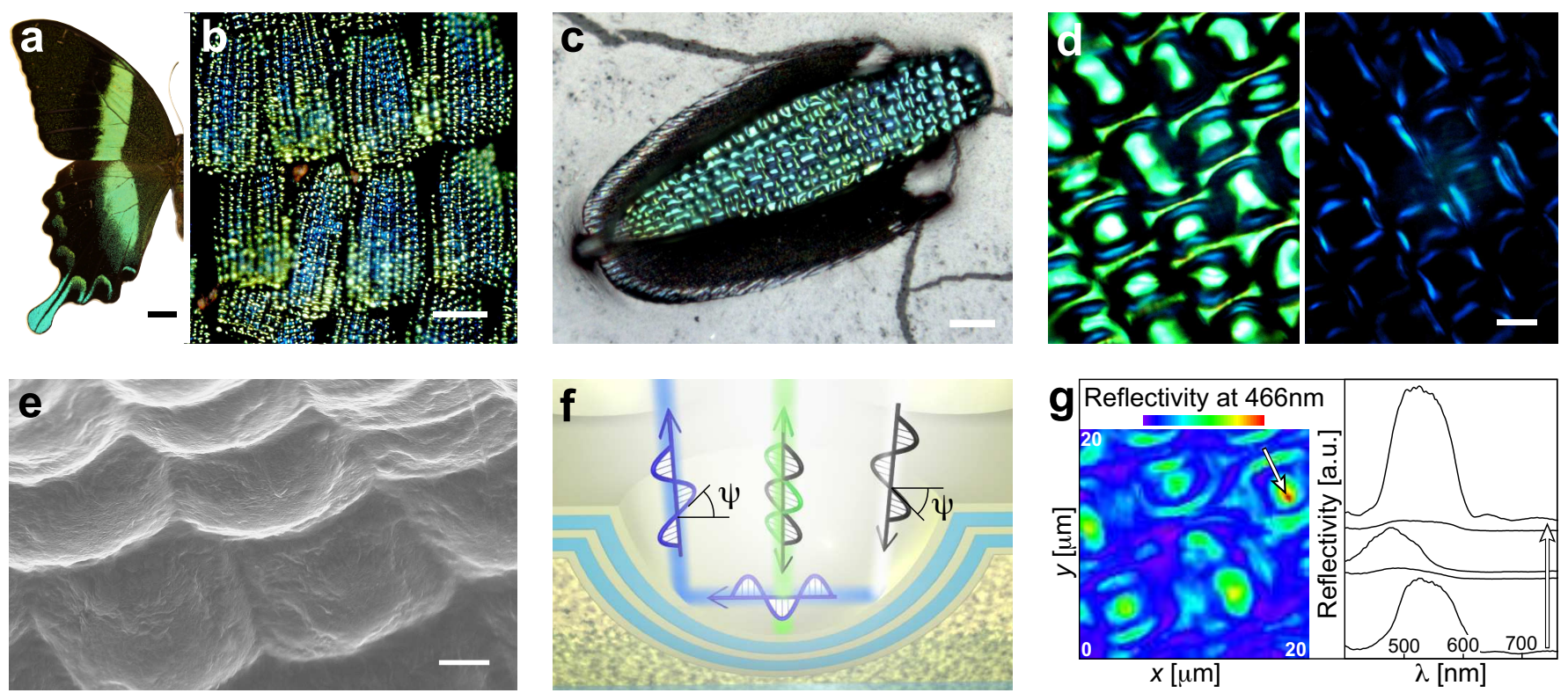

Figure 1 | Natural photonic structure. The bright green on Papilio blumei's wings (a, scale bar $1 \mathrm{~cm}$ ) results from a colour mixing ${ }^{7}$ of blue and yellow-green light reflected from different wing scale regions (b, scale bar $\left.100 \mu \mathrm{m}\right)$. Papilio blumei has two types of scales: The first kind provides the colour while the second kind, lying under the first, absorbs the transmitted light, preventing it from being backscattered, thus assuring the purity of the reflected colours (c, scale bar $20 \mu \mathrm{m}$ ). The surface of a reflecting wing scale (optical micrograph in $\mathbf{d}$ and scanning electron micrograph in e, scale bars $5 \mu \mathrm{m}$ and $2 \mu \mathrm{m}$ ) is covered with concavities of about $5-10 \mu \mathrm{m}$ diameter arranged in ordered lines along the scale. These concavities are clad with a multilayer reflecting yellow-green light in the concavity centres whereas light reflected from the edges is blue ${ }^{8}$ (d, left). By observing the scales in a light microscope with crossed polarisers, light reflected from the concavity centres is extinguished (d, right), while blue light from four segments of the concavity edges is detected, because of a polarisation rotation caused by its double reflection inside the concavity. This polarisation rotation results partly from the out-of-plane reflections at each interface geometrically rotating polarised light by an angle of $2 \psi^{12}$ (f). Spectral maps of some of the concavities confirm the optical anisotropy of the scale surface for unpolarised light (g, left). Corresponding spectra (g, right), taken along the white arrow, displayed in the spectral map, show the shift in reflectance peak from green at the very edge of the concavity to blue close to the perimeter and back to green at the centre of the concavity.

tilayer stack, reducing specular reflections and unwanted destructive interferences which otherwise severely limit the optical performance.

Optical analysis of the natural butterfly structure and the artificial mimic is performed using a microspectroscopic setup that allows the collection of spectral data in a sample region of less than $1 \mu \mathrm{m}$ in diameter. The two-dimensional translation of the sample under the objective of the microscope enables the acquisition of spectral maps across several concavities.

The artificial mimic presented in this work is composed of multilayer concavities of $\approx 4.5 \mu \mathrm{m}$ in diameter and $\approx 2.3 \mu \mathrm{m}$ in height (Fig. 3a,b). The multilayer consists of 11 alternating $(57 \pm 4) \mathrm{nm}$ thick titania and $(82 \pm 4) \mathrm{nm}$ thick alumina layers. These particular layer thicknesses were chosen in the attempt to create a quarter wave stack with stop-band centre wavelength in the green-yellow spectral range (around $550 \mathrm{~nm}$ ) to match the reflectance band of the natural Papilio structure closely. The refractive indices of the titania $\left(n_{\mathrm{TiO}_{2}}=2.5 \pm 0.1\right)$ and alumina $\left(n_{\mathrm{Al}_{2} \mathrm{O}_{3}}=1.7 \pm 0.1\right)$ layers were measured by ellipsometry. Both materials show no significant optical adsorption in thin films. The peak reflectance wavelength of the multilayer at $\approx 550 \mathrm{~nm}$ normal light incidence (i.e. in the centres of the cavity) corresponds well to theoretical predictions. Eleven layers are sufficient to achieve a peak reflectivity of more than $95 \%$. These multilayer coated structures immediately display iridescent colours (Fig. 3c,d).

The bandwidth of the butterfly scale reflectance peak $\Delta \lambda \approx 105 \mathrm{~nm}$ is slightly larger, than expected for a flat multilayer $\Delta \lambda_{\mathrm{th}}=\frac{2}{\pi} \bar{\lambda} \frac{\Delta \mathrm{n}}{\bar{n}} \approx 74 \mathrm{~nm}$ where $\bar{\lambda}$ is the peak-centre wavelength, $\Delta \mathrm{n}$ the refractive index difference, and $\bar{n}$ the average refractive index of the multilayer materials. For the original butterfly structure, the refractive index of the solid cuticle layers is taken as $\mathrm{n}_{\text {cuticle }}=1.56^{13}$. A fit to the reflection data yielded a refractive index of $\approx 1.25$ for the cuticle-pillar-supported air layers. In comparison, the reflectance peak width of the artificial structure $\Delta \lambda \approx 140 \mathrm{~nm}\left(\Delta \lambda_{\mathrm{th}}=134 \mathrm{~nm}\right)$ is broader because of the higher ratio $\frac{\Delta \mathrm{n}}{\overline{\mathrm{n}}}$ of $\mathrm{TiO}_{2}$ and $\mathrm{Al}_{2} \mathrm{O}_{3}$.

Optical microscopy images show a similar colour variation from the concavity centres to their edges for both 


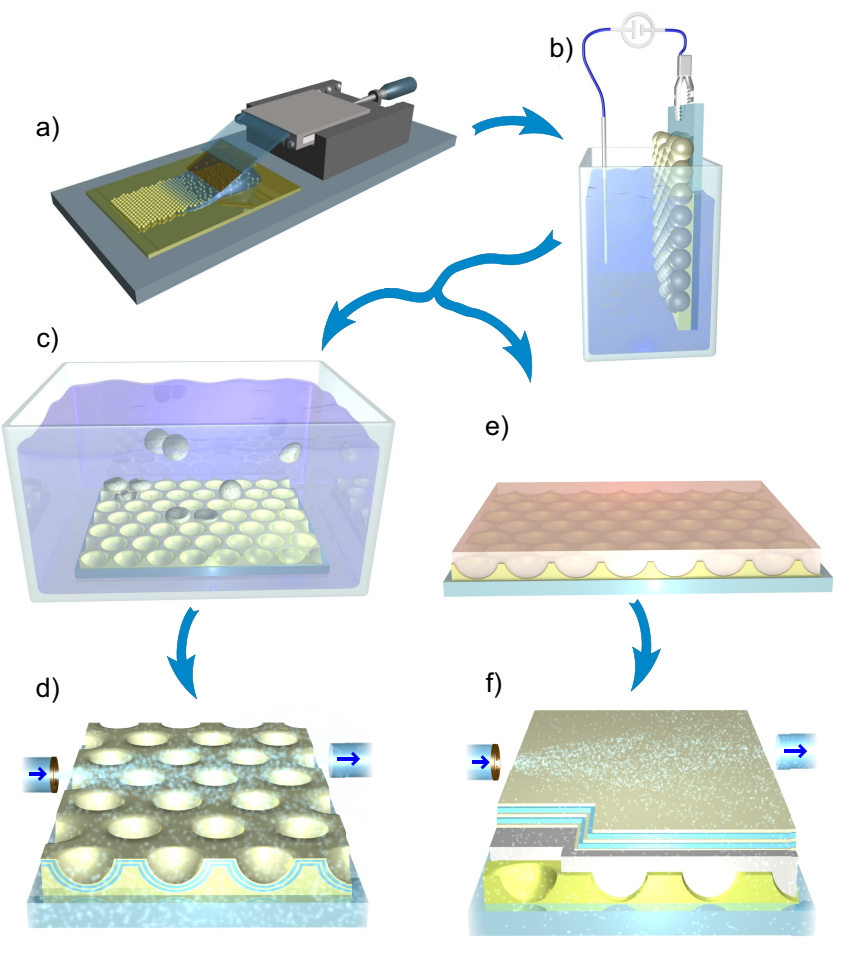

Figure 2 Sample fabrication. a, Deposition of polystyrene colloids on a gold-coated silicon substrate. $\mathbf{b}$, Growth of platinum or gold in the interstices of the colloidal array via electro-plating. The metal deposition is terminated when the thickness of the deposited film equals the microsphere radius. c, Removal of the polystyrene spheres from the substrate by ultra-sonication in acetone. d, Sputtering of a thin carbon film and atomic layer deposition of a stack of 11 alternating $\mathrm{TiO}_{2}$ and $\mathrm{Al}_{2} \mathrm{O}_{3}$ layers (arrows indicate the precursor gas flow). In a second route $\mathbf{e}$, the colloids are molten to cover the cavities with a homogeneous film, which is, $\mathbf{f}$, covered by a $\mathrm{TiO}_{2}-\mathrm{Al}_{2} \mathrm{O}_{3}$ multilayer.

the natural structure and the replica (comparison of Fig. 3e and Fig. 1d). This is confirmed by the peak shift in the corresponding spectra (Fig. 3g, left). The experimental data is well described by results of theoretical modelling of the multilayer structure. The anisotropic reflectance of the sample is clearly visible in the spectral maps (Fig. $3 f$, left).

The observation of the artificial mimic between crossed polarisers leads to a similar effect described above for the butterfly structure. Only light incident onto four segments of the concavity edges is detected. The local surface normal of $\approx 45^{\circ}$ gives rise to a double reflection at opposing cavity walls causing a polarisation rotation (Fig. 3f, right). As expected, this light is blue-shifted with respect to the light reflected from the centres of the concavities. The artificial mimic therefore displays the same optical characteristics as the natural Papilio blumei wing scale structure.

In addition, a new feature arises from the double reflec- tion of a high refractive index contrast dielectric stack, which is the two peak reflectivity seen in Fig. 3 when the sample is placed between crossed polarisers. The origin of this spectrum goes beyond the purely geometrical polarisation conversion, but depends on the different complex reflection coefficients, $r_{\mathrm{s}}, r_{\mathrm{p}}$, for s- and p-polarised light reflected from the multilayer. Both the magnitude and phase of $r_{\mathrm{s}}$ and $r_{\mathrm{p}}$ differ at different wavelengths relative to the reflection stop-band, with a spectrally-narrower stop band for p-polarised light. Full modelling of the multi-bounce reflectivity shows that the double-bounce cannot produce a double-peak feature. However, retroreflection via a triple bounce can also occur ${ }^{12}$ when light hits the outer edge of a concavity at an angle of $\approx 60^{\circ}$. For this triple bounce, the combination of geometrical polarisation, relative-phase-shift-induced ellipticity and polarisation conversion based on a reflectivity difference leads to the observed double peak structure. The reflection data is well fit by superposition of reflections from both double and triple bounces (red curves in Fig. 3g, right). Although the triple bounce reflection can also take place in the natural Papilio scale concavities, the double peak feature is not experimentally discernible for two reasons: (1) Optical modelling shows only a shallow dip in the triple bounce reflection peak for the butterfly's air-cuticle multilayer concavities. (2) Naturally occurring variations in concavity size and orientation lead to a mixing of the triple and double bounce signal, shadowing the faint double peak feature entirely.

The angular change in peak reflectance wavelength, $\lambda$, follows the simple relation

$$
m \frac{\lambda}{2}=d_{1} \sqrt{n_{1}^{2}-\sin ^{2} \theta_{0}}+d_{2} \sqrt{n_{2}^{2}-\sin ^{2} \theta_{0}}
$$

where $n_{1}, n_{2}, d_{1}$, and $d_{2}$ denote the refractive indices and thicknesses of the two different multilayer materials, $\theta_{0}$ is the light incidence angle and $m$ is a positive integer. Using titania-alumina multilayers, peak wavelength shifts from the $45^{\circ}$ double reflection of $35 \mathrm{~nm}$ can be achieved, compared to $60 \mathrm{~nm}$ for the natural butterfly structure. The use of a material with a smaller refractive index $n<n_{\mathrm{Al}_{2} \mathrm{O}_{3}}$ for the low refractive index component in the multilayer stack will increase this peak wavelength shift.

Alternatively, using a combination of high refractive index dielectrics with lower refractive index contrast (for instance zinc oxide and titanium oxide, $\frac{\Delta n}{\bar{n}} \approx 0.19$ as opposed to $\frac{\Delta n}{\bar{n}} \approx 0.39$ of $\mathrm{Al}_{2} \mathrm{O}_{3}$ and $\mathrm{TiO}_{2}$ ) for the multilayer coating would result in smaller reflectance band widths and consequently cause a more perceivable colour hue change across the concavities. High reflectivity with materials of low refractive index contrast involves Bragg mirrors with a larger number of layers, however.

We instead introduce a third alternative for the enhancement of a structural colour effect. A small simplification in the manufacture strategy described above leads to a pronounced colour variation (Fig. 4). Start- 

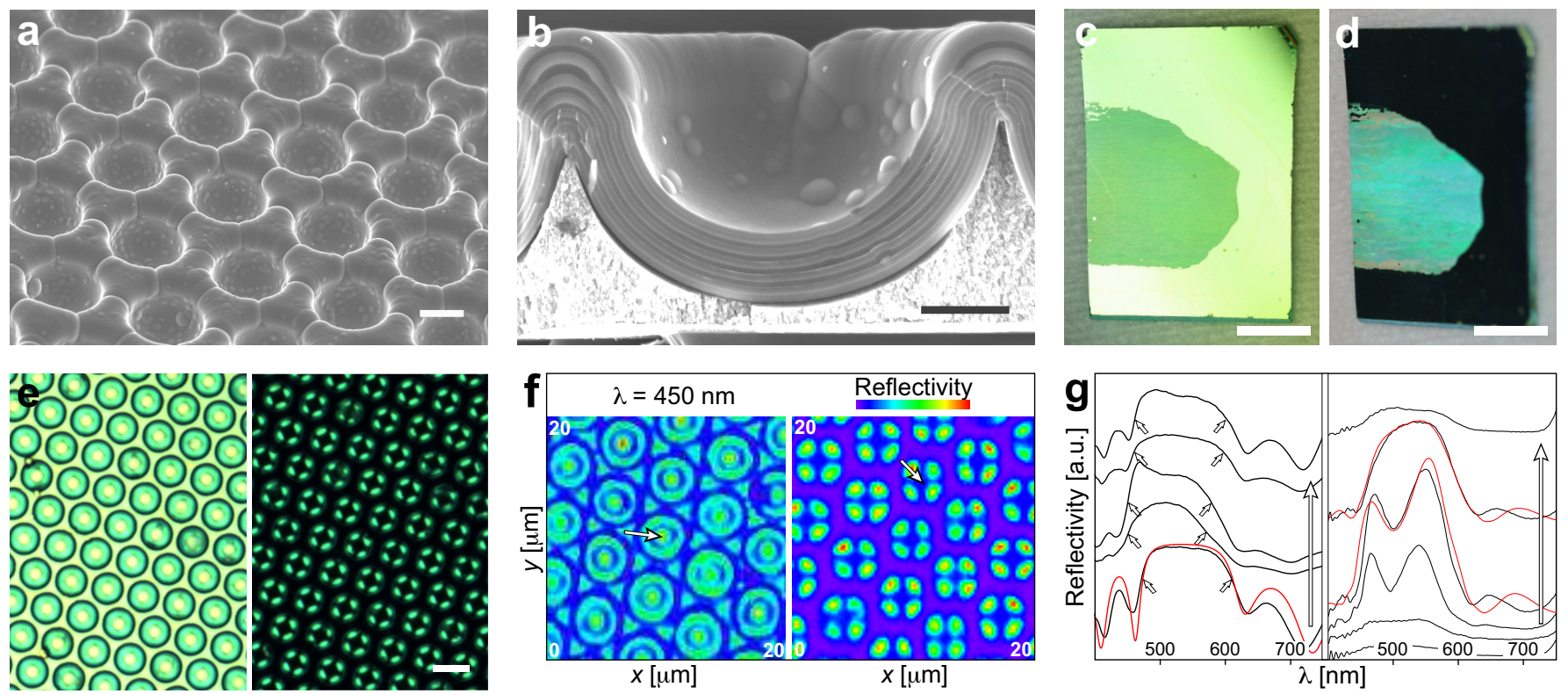

Figure $3 \mid$ An artificial optical mimic. a, b, SEM images of concavities which are covered by a conformal multilayer stack of 11 alternating layers of titania and alumina: a top view, $2 \mu \mathrm{m}$, b cross-section, $1 \mu \mathrm{m}$. c. At perpendicular light incidence the artificial replica appears green, while it, $\mathbf{d}$, reflects blue at grazing incidence, showing some iridescence (scale bars $5 \mathrm{~mm}$ ). e, Under a light microscope, the concavity edges appear turquoise, while the centres and interstitial regions are yellow (left); between crossed polarisers only the concavity edges are visible (right, scale bar $5 \mu \mathrm{m}$ ). f, The spectral maps for $\lambda=450 \mathrm{~nm}$ visualise the anisotropic reflectivity of the concavities for unpolarised light (left) and between crossed polarisers (right). $\mathbf{g}$ shows reflectivity along the paths indicated by arrows in the two frames in $\mathbf{f}$, respectively. $\mathbf{g}$, left: The reflectance peak-shift across the concavities is clearly visible. The small arrows indicate the reflectance band edges. The red curve represents the predicted reflectance of the multilayer for normal light incidence. g, right: Light transmitted through crossed polarisers has undergone a polarisation rotation via a double or triple bounce. The double bounce results in a single reflection peak and a triple bounce induces a double peak feature. The red curves model the reflection as a superposition of double and triple bounce.

ing from a self-organised colloidal monolayer and electrochemical gold deposition, a multilayer is deposited. However, instead of removing the colloids prior to ALD deposition, the sample is annealed at $200^{\circ} \mathrm{C}$, the colloids melt and form a continuous film of polymer, entirely filling and covering the concavities, thereby creating a flat surface. The ALD deposition of a planar titania-alumina multilayer on top of the polymer film results in a sample with periodically shaped resonant cavities. When seen in specular reflection the surface appears bright blue. Observed in back reflection for non-normal light incidence it is strikingly red.

Visual information can be encoded into this photonic structure by photolithographically creating an arbitrary pattern in $\mathrm{a} \approx 200 \mathrm{~nm}$ thick resist layer on the conducting surface prior to deposition of the colloidal template. The thin photoresist pattern does not influence the assembly of the colloids but prevents gold electro-deposition. Consequently, the concave micro-mirrors are formed only in resist-free areas thereby creating a picture, the colour of which varies dramatically with observation and light incidence angle.

The modified photonic structure has an optical signature which is very similar to that of Fig. 3 in terms of optical anisotropy when scanning across the concavities and the behaviour in polarised light. The important difference to the conformal multilayer concavities is that the two predominant colours that are reflected on the micro-scale are not correlated by Eq. 1. The underlying mechanism for colour creation is different. While most of the incident light with wavelengths in the blue part of the visible spectrum is directly reflected by the upper multilayer and therefore does not enter the polymer cavity, light in the red part of the spectrum is transmitted by the multilayer and enters the cavities. There, most of it is reflected back in the direction of incidence by the underlying array of concave gold micro-mirrors. As expected, a blue-shift in the reflection band of the multilayer is observed for increasing incidence angles, resulting in a variation of the back-reflected colour from red to orange. Only a small fraction of red light is scattered from the inter-cavity ridges into a wide angular range.

Additional cavity resonances in the red, particularly for light reflected off the concavity centres or the interstitial regions between adjacent concavities contribute to the colour signature of the patterned device by a subtle modification in colour hue. For the thicker concavity centres, these resonances are closely spaced in wavelength, 

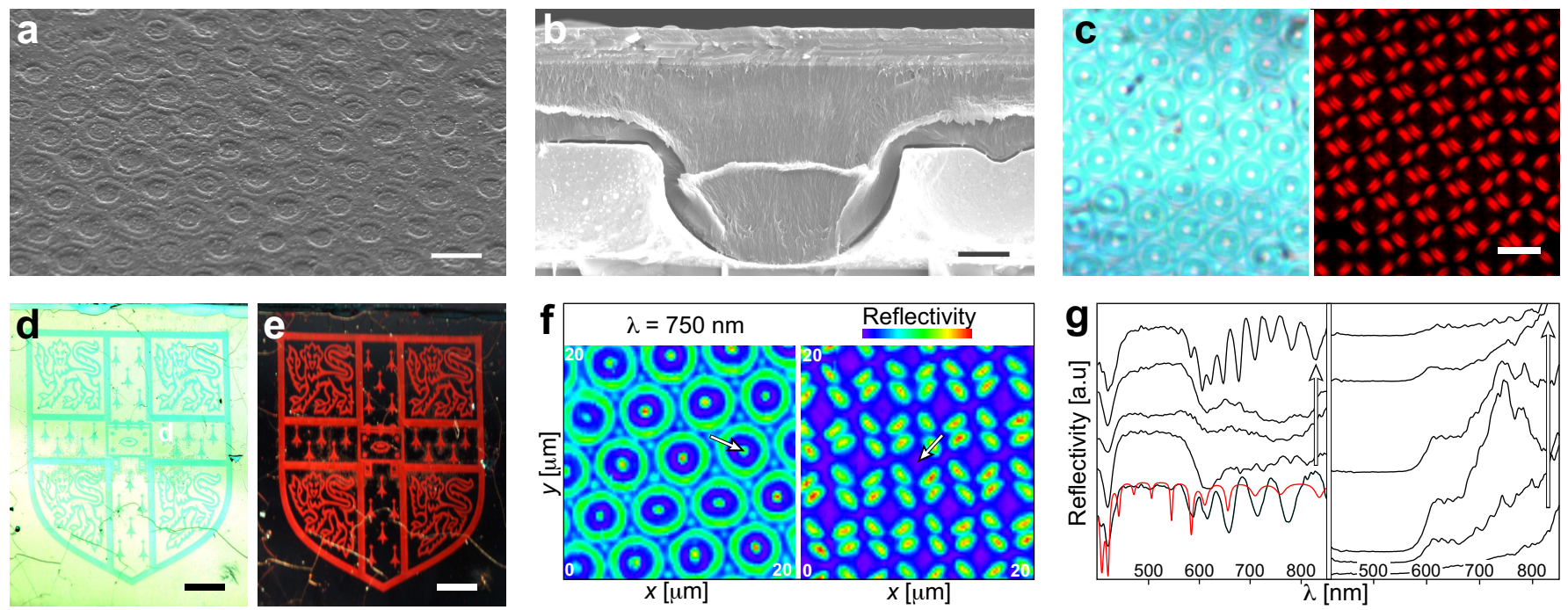

Figure 4 | Modified mimic with enhanced optical performance. a, b, SEM images of melted colloidal spheres which are embedded in $5 \mu \mathrm{m}$ wide gold concavities and covered by a planar multilayer stack of 11 alternating layers of titania and alumina (a top view, $5 \mu \mathrm{m}$, b cross-section, $1 \mu \mathrm{m}$ ). c, Under unpolarised light, the edges of the concavities appear blue, while the centres and interstitial regions are reflecting in a broad spectral range (left). Between crossed polarisers only reflected red light is detected (right, scale bar $2 \mu \mathrm{m}$ ). Samples viewed in d, direct specular reflection and in e, retro-reflection (scale bar $5 \mathrm{~mm}$ ) show a striking change in colour from blue to red. $\mathbf{f}$, The anisotropic concavity reflectivity is clearly visible in the spectral maps for unpolarised $\lambda=750 \mathrm{~nm}$ illumination (left) and between crossed polarisers (right). g, Spectra from distinct points on these spectral maps along the white arrows in $\mathbf{f}$. Left: change in unpolarised reflectivity varying from the concavity border to its centre. The red line shows the calculated reflectance curve of the multilayer structure for the interstitial areas. The reflectance peak resulting from the multilayer does not shift significantly across the cavity, but strong resonances in the red are observed in the concavity centres and in the interstitial areas. $\mathbf{g}$, right: concavity reflectivity between crossed polarisers. Only red light retro-reflected from the inclined edges accompanied by a polarisation rotation is detected.

compared to the interstitial regions. The experimentally observed spectra match well with theoretical models for layer thicknesses of $\approx 3850 \mathrm{~nm}$ in the concavity centres and $\approx 1865 \mathrm{~nm}$ in the interstitial regions (Fig. $4 \mathrm{~g}$ ).

The sample of Fig. 4 exhibits a pronounced variation from pale blue in specular reflection via red in all other directions to a particularly brilliant red in retro-reflection. By changing the multilayer spacing, it is possible to tune the colour seen in specular reflection across the whole visible range, accompanied by the complementary colour in retro-reflection. A change in thickness of the polymer cavity results in a variation of the resonant wavelengths of the light that is transmitted through the top multilayer, modifying the colour seen in back reflection for non-normal light incidence.

In summary, the intricate surface structure of Papilio butterflies was replicated in five simple steps by replicating a colloidal monolayer into an inorganic optical structure. A variation of concavity height gives rise to a colour appearance mimicking either the single coloured Papilio ulysses, (suppressing double and triple bounce when the concavity walls are low enough) or the colour mixing of Papilio palinurus or Papilio blumei ${ }^{8}$. Square-centimetre sized samples were fabricated in a facile and scaleable approach. The manufactured mimics differ in important ways from the natural counterparts: (1) by using solid inorganic materials instead of perforated cuticle lamella, the optical structure is much less fragile and a much wider range in refractive index contrast can be achieved. (2) The in-plane hexagonal symmetry of the concavities (in contrast to the quasi-1D alignment of the concavities of a Papilio wing scale) can give rise to additional grating interferences, the extent of which can be controlled by varying the conditions under which the colloidal monolayer is deposited. Furthermore, we have demonstrated that a small variation of the natural design principle allows the creation of a striking colour separation effect. Rather than the juxtaposition of two colours in Papilio butterflies, adjustable switching for any colour and its complementary hue can be achieved. Square-centimetre sized patterns and pictures with micro-scale resolution were encoded in the photonic structure, rendering this approach versatile for applications in the fields of security labelling or the manufacture of dynamic and vivid paints and coatings. This striking effect may also be used as a signalling cue in other patterned insects and should be a focus of future work. 
1. Vukusic P. \& Sambles J.R. Photonic structures in biology. Nature 424, 852-855 (2003).

2. Land M.F. The physics and biology of animal reflectors. Prog. Biophys. Mol. Biol. 24, 75-106 (1972).

3. Doucet S. \& Meadows M. Iridescence: A functional perspective. J. R. Soc. Interface 6, S115-S132 (2009).

4. Parker A.R. \& Townley H.E. Biomimetics of photonic nanostructures. Nature Nanotech 2, 347-353 (2007).

5. Watanabe K., Hoshino T., Kanda K., Haruyama Y. \& Matsui S. Brilliant blue observation from a Morphobutterfly-scale quasi-structure. Jpn. J. Appl. Phys. 44, L48-L50 (2005).

6. Huang J., Wang, X. \& Wang Z.L. Controlled replication of butterfly wings for achieving tunable photonic properties. Nano Lett. 6, 2325-2331, (2006).

7. Vukusic P., Sambles R.J. \& Lawrence C.R. Colour mixing in wing scales of a butterfly. Nature 404, 457 (2000).

8. Vukusic P., Sambles R.J., Lawrence C.R. \& Wakely G. Sculpted-multilayer optical effects in two species of $P a$ pilio butterfly. Appl. Opt. 40, 1116-1125 (2001).

9. Gaillot D.P., Deparis O., Welch V., Wagner B.K., Vigneron J.P. \& Summers C.J. Composite organic-inorganic butterfly scales: Production of photonic structures with atomic layer deposition. Phys. Rev. E 78, 031922 (2008).

10. Chen Y., Gu J., Zhu S., Fan T., Zhang D. \& Guo Q. Iridescent large-area $\mathrm{ZrO}_{2}$ photonic crystals using butterfly as templates. Appl. Phys. Lett. 94, 053901 (2009).

11. Zhu S., Zhang D., Chen Z., Gu J., Li W., Jiang H. \& Zhou G. A simple and effective approach towards biomimetic replication of photonic structures from butterfly wings. Nanotechnology 20, 315303 (2009).

12. Coyle S., Prakash G.V., Baumberg J.J., Abdelsalam M. \& Bartlett P.N. Spherical micro-mirrors from templated self-assembly: Geometric reflectivity on the micron scale. Appl. Phys. Lett. 83, 767-769 (2003).

13. Vukusic P., Sambles J.R., Lawrence C.R. \& Wootton R.J. Quantified interference and diffraction in single Morpho butterfly scales. Proc. R. Soc. London Ser. B 266, 14031411 (1999).

14. Bartlett P.N., Birkin P. R. \& Ghanem M. A. Electrochemical deposition of macroporous platinum, palladium and cobalt films using polystyrene latex sphere templates. Chem. Commun., 1671-1672 (2000).

15. Braun P. V. \& Wiltzius P. Macroporous materials - electrochemically grown photonic crystals. Curr. Opin. Colloid Interface Sci. 7, 116-123 (2002).

16. Wijnhoven J.E.G.J., Zevenhuizen S. J. M., Hendriks M.
A., Vanmaekelbergh D., Kelly J. J. \& Vos W. L. Electrochemical assembly of ordered macropores in gold. $A d v$. Mater. 12, 888-890 (2000).

17. Puurunen R.L. Surface chemistry of atomic layer deposition: A case study for the trimethylaluminum/water process. J. Appl. Phys. 97, 121301 (2005).

\section{Acknowledgements}

Financial support from EPSRC (EP/G060649/1, $\mathrm{EP} / \mathrm{E} 040241$, EP/C511786/1) is gratefully acknowledged. M.K. acknowledges support from DAAD (German Academic Exchange Service) and the Cambridge Newton Trust.

\section{Author contributions}

P.V. and M.K. performed the studies of the natural photonic structure. M.K., M.S., P.S. and U.S. conceived and designed the artificial mimics. M.S. provided the colloidal templates. P.S. and S.M. performed the electro-deposition. P.S. produced the photolithographic resist pattern for the modified mimic. M.K. and P.S. realised the atomic layer deposition. M.K. characterised the optical performance and the topology of the samples. F.H. and J.B. provided the algorithms necessary to perform the optical measurements and to create the spectral maps. M.K., J.B., U.S. and P.V. analysed the data. M.K., U.S. and J.B. wrote the paper. All authors discussed the results and commented on the manuscript.

\section{Additional information}

Supplementary information accompanies this paper at www.nature.com/naturenanotechnology. Reprints and permission information is available online at http://npg.nature.com/reprintsandpermissions/.

Correspondence and requests for materials should be addressed to J.J.B. and U.S. 